\title{
Identification of five novel genetic loci related to facial morphology by genome- wide association studies
}

\author{
Seongwon Cha ${ }^{1 \dagger}$, Ji Eun Lim² ${ }^{2 \dagger}$, Ah Yeon Park ${ }^{3}$, Jun-Hyeong Do', Si Woo Lee ${ }^{1}$, Chol Shin ${ }^{4}$, Nam Han Cho ${ }^{5}$, \\ Ji-One Kang${ }^{2}$, Jeong Min Nam², Jong-Sik Kim ${ }^{6}$, Kwang-Man Woo ${ }^{6}$, Seung-Hwan Lee ${ }^{6}$, Jong Yeol Kim \\ and Bermseok Oh²
}

\begin{abstract}
Background: Face morphology is strongly determined by genetic factors. However, only a small number of genes related to face morphology have been identified to date. Here, we performed a two-stage genome-wide association study (GWAS) of 85 face morphological traits in 7569 Koreans (5643 in the discovery set and 1926 in the replication set).

Results: In this study, we analyzed 85 facial traits, including facial angles. After discovery GWAS, 128 single nucleotide polymorphisms (SNPs) showing an association of $P<5 \times 10^{-6}$ were selected to determine the replication of the associations, and meta-analysis of discovery GWAS and the replication analysis resulted in five genome-wide significant loci. The OSR1-WDR35 [rs7567283, G allele, beta $(\mathrm{se})=-0.536(0.096), P=2.75 \times 10^{-8}$ ] locus was associated with the facial frontal contour; the HOXD1-MTX2 [rs970797, A allele, beta (se) $=0.015(0.003), P=3.97 \times 10^{-9}$ ] and WDR27 [rs3736712, C allele, beta $(\mathrm{se})=0.293(0.048), P=8.44 \times 10^{-10} \mathrm{]}$ loci were associated with eye shape; and the SOX9 [rs2193054, C allele, beta (se) (In-transformed) $=-0.007(0.001), P=6.17 \times 10^{-17}$ ] and DHX35 [rs2206437, A allele, beta $(\mathrm{se})=-0.283(0.047), P=1.61 \times 10^{-9}$ ] loci were associated with nose shape. WDR35 and SOX9 were related to known craniofacial malformations, i.e., cranioectodermal dysplasia 2 and campomelic dysplasia, respectively. In addition, we found three independent association signals in the SOX9 locus, and six known loci for nose size and shape were replicated in this study population. Interestingly, four SNPs within these five face morphology-related loci showed discrepancies in allele frequencies among ethnic groups.
\end{abstract}

Conclusions: We identified five novel face morphology loci that were associated with facial frontal contour, nose shape, and eye shape. Our findings provide useful genetic information for the determination of face morphology.

Keywords: Face morphology, GWAS, Korean, OSR1-WDR35, HOXD1-MTX2, WDR27, SOX9, DHX35

\section{Background}

Face morphology is an important feature for both craniofacial clinics and forensic science. Clinically, craniofacial deformities comprise over half of all congenital malformations [1], and affected patients suffer not only from clinical syndromes but also social maladjustment $[2,3]$. In forensic science, genetic information is considered to be important evidence, providing data regarding

\footnotetext{
*Correspondence: ohbs@khu.ac.kr

t'Seongwon Cha and Ji Eun Lim contributed equally to this work.

Jong Yeol Kim and Bermseok Oh jointly supervised this work.

${ }^{2}$ Department of Biochemistry and Molecular Biology, School of Medicine,

Kyung Hee University, Seoul 02447, Republic of Korea

Full list of author information is available at the end of the article
}

personal identification and externally visible characteristics such as iris and hair color $[4,5]$. Recently, advanced high-resolution three-dimensional imaging techniques that use face morphology as a statistical value have opened up new possibilities of genetically aided facial modeling technology combined with more available DNA information $[6,7]$.

The heritability of craniofacial traits has been reported to be 0.8 based on analysis of the lateral cephalogram from X-ray profiles of parents and their offspring [8] or $0.41-0.86$ based on twin studies [9], suggesting that face morphology is more strongly determined by genetic factors than environmental factors. The process of face

(C) The Author(s). 2018 Open Access This article is distributed under the terms of the Creative Commons Attribution 4.0 International License (http://creativecommons.org/licenses/by/4.0/), which permits unrestricted use, distribution, and 
formation is an evolutionarily conserved, precisely orchestrated process involving the cellular migration, interaction, proliferation, and differentiation of diverse tissue cells [10]. Studies using human subjects and animal models with congenital craniofacial malformations have explored the genetic factors affecting face formation. Consequently, multiple signaling pathways have been reported to be important during embryonic craniofacial morphogenesis, including bone morphogenic protein, sonic hedgehog, fibroblast growth factor, growth hormone receptor, and Wnt/ $\beta$-catenin pathways [11].

Paternoster et al. [12] conducted a genome-wide association study (GWAS) and found that a genetic variant of the PAX3 locus was associated with nose shape. Liu et al. [13] confirmed the association of the PAX3 locus in European subjects, and further identified four novel genetic variants affecting face morphology close to the genes PRDM16, TP63, C5orf50, and COL17A1. More recently, Adhikari et al. [14] identified five more genetic variants related to nose shape close to the genes $D C H S 2$, RUNX2, GLI3, PAX1, and EDAR. Shaffer et al. [15] identified seven additional genetic variants for face traits such as facial width and depth, and nose shape. Cole et al. [16] identified two additional genetic variants of SCHIP1 and PDE8A associated with facial size, and Lee et al. [17] identified two additional genetic variants of FREM1 and PARK2 associated with face shape.

Despite these studies, our current understanding of face morphology falls far short of expectations in craniofacial medicine and forensic science. Therefore, the discovery of more genetic variants using a large-scale face morphology GWAS may contribute to identification of the etiology of human craniofacial malformations and externally visible characteristics. Therefore, in this study, we aimed to identify genetic factors associated with face morphology in a large cohort of 7569 samples from the Korean population (East Asians). To the best of our knowledge, this study represents the first GWAS on facial morphology in the Korean population.

\section{Results}

\section{Characteristics of facial traits and their heritability}

For a comprehensive investigation of face morphology, a total of 85 facial parameters were selected from both frontal and lateral pictures of the individual subjects, including 17 frontal, 11 forehead, 13 eye, 32 upper eyelid, 11 nose, and two mouth features (Table 1). Facial traits were delineated by distance, distance ratio, angle, area, and curvature (eyelid) from 23 frontal and seven lateral face points, which were automatically extracted from each picture using in-house developed software (Fig. 1) [18].

We first analyzed the correlation between body mass index (BMI) and facial traits. As shown in
Additional file 1: Table S1, the correlation was the greatest for facial width traits, including facial base width, lower facial width, upper facial width, and middle facial width $(r=0.355-0.487)$. We also analyzed the correlation between sex or age and facial traits. As shown in Additional file 1: Table S1, the correlations of sex were the greatest for profile nasal area $(r=-0.542)$ and upper facial area $(r=-0.516)$, and the correlations of age were the greatest for eye traits such as tangent line angle of er7 or el7 ( $r=-0.306$ to -0.326$)$ and palpebral fissure heights $(r=-0.301$ to -0.312$)$. As expected, significant correlations were observed between similar traits under the criterion of a Bonferroni-adjusted $P$-value threshold for significance of $1 \times 10^{-5}$ (Additional file 2: Table S2). The correlation coefficients $(r)$ between facial width traits, including facial base width, lower facial width, upper facial width, and middle facial width, ranged from 0.643 to 0.928 . Among the nose traits, the profile nasal area was correlated to nasal bridge depth $(r=0.573)$ and to nasal tip protrusion $(r=0.771)$.

We also estimated narrow-sense heritability for the facial traits using the GCTA program, and found moderate values for most traits (Additional file 3: Table S3). The highest heritability was detected in nasal tip protrusion (0.417), nasolabial angle (0.365), and upper lip thickness (0.299 and 0.344, right and left respectively).

\section{Discovery GWAS}

We performed a discovery GWAS (Phase $1 ; n=5643$ ) and a replication analysis (Phase $2 ; n=1926$ ) for the 85 facial traits, as shown in Additional file 4: Figure S1. The characteristics of the participants in the two phases are described in Additional file 5: Table S4.

In total, 311,944 single nucleotide polymorphisms (SNPs) were examined in the linear regression model as independent variables of facial traits, controlled for age, sex, and BMI as covariates. The Q-Q plots of the discovery GWAS for each face trait are shown in Additional file 6: Figure S2. The GWAS results are also displayed as $-\log _{10}(P)$ values against the chromosomal position on Manhattan plots in Additional file 7: Figure S3.

Among the 85 facial traits, seven traits, including two eye-related traits [eye tail length (psR-exR) and tangent line angle of el3 ((A) Tan_el3)] and five nose-related traits [nasal bridge depth $((\mathrm{H}) \mathrm{n}$-prn], nasal tip protrusion $[(\mathrm{H})$ prn-sn], profile nasal area [(AR) n-prn-sn], nasolabial angle [(A) prn-sn], and profile nasal angle [(A) n-prn-sn)] showed genetic associations at the genome-wide significance level $\left(P<5 \times 10^{-8}\right)$ in the discovery GWAS (Additional file 8: Table S5). SOX9, $T B X 3-M E D 13 L$, and $V P S 13 B$ loci met the genome-wide significance level for nose-related traits, and WDR27 and HOXD-MTX2 loci met the genome-wide significance level for eye shape. 
Table 1 Eighty-five facial traits from 23 frontal, 7 profile, and 16 upper eyelid points

\begin{tabular}{|c|c|c|c|c|c|}
\hline \multicolumn{3}{|c|}{ Size-related variables } & \multicolumn{3}{|c|}{ Shape-related variables } \\
\hline & Abbreviation & Description & & Abbreviation & Description \\
\hline Face shape & (17 traits) & & & & \\
\hline \multirow[t]{4}{*}{ Width $^{a}$} & zyR-zyL & Facial base width & Ratio & zyR-zyL/goR -goL & Facial width ratio of base to chin \\
\hline & goR -goL & Lower facial width & & zyR-zyL/n-sto & Facial ratio of base width to height \\
\hline & obsR-obsL & Upper facial width & & goR -goL/n-sto & Facial ratio of chin width to height \\
\hline & obiR-obiL & Middle facial width & & & \\
\hline \multirow[t]{2}{*}{ Height $^{b}$} & sn-sto & Upper lip height & Angle ${ }^{c}$ & (A) enR-exR-goR & Right facial angle of en-ex-go \\
\hline & n-sto & Facial height & & (A) enL-exL-goL & Left facial angle of en-ex-go \\
\hline \multirow[t]{4}{*}{ Area } & (AR) obsR-obsL-obiR-obiL & Upper facial area & & (A) psR-exR-goR & Right facial angle of ps-ex-go \\
\hline & (AR) obiR-obiL-goR -goL & Lower facial area & & (A) psL-exL-goL & Left facial angle of ps-ex-go \\
\hline & & & & (A) enR-psR-goR & Right facial angle of en-ps-go \\
\hline & & & & (A) enL-psL-goL & Left facial angle of en-ps-go \\
\hline Forehead & (10 traits) & & & & \\
\hline \multirow[t]{6}{*}{ Height $^{b}$} & (V) tr-o & Forehead height & Angle ${ }^{c}$ & (A) $m$-tr & Upper forehead slant angle \\
\hline & $(V)$ m-o & Lower forehead height & & (A) o-n & Brow ridge protrusion angle \\
\hline & (V) tr-m & Upper forehead height & Ratio & mtro-o/t-o & Metopion position ratio \\
\hline & $(V)$ o-n & Brow ridge height & Depth & $(\mathrm{H}) \mathrm{tr}-\mathrm{m}$ & Upper forehead slant depth \\
\hline & & & & $(H)$ o-n & Brow ridge protrusion \\
\hline & & & & m-mtro & Metopion eminence depth \\
\hline Eye & (15 traits) & & & & \\
\hline \multirow[t]{4}{*}{ Width $^{a}$} & enR-enL & Intercanthal width & Angle ${ }^{c}$ & (A) exR-psR & Right eye angle of ex-ps \\
\hline & exR-exL & Outercanthal width & & (A) exL-psL & Left eye angle of ex-ps \\
\hline & & & & (A) enR-psR & Right eye angle of en-ps \\
\hline & & & & (A) enL-psL & Left eye angle of en-ps \\
\hline \multirow[t]{2}{*}{ Height $^{\mathrm{b}}$} & psR-piR & Right palpebral fissure height & & (A) enR-psR-exR & Right eye angle of en-ps-ex \\
\hline & psL-piL & Left palpebral fissure height & & (A) enL-psL-exL & Left eye angle of en-ps-ex \\
\hline \multirow[t]{3}{*}{ Length } & enR-exR & Right palpebral fissure length & Length & psR-exR & Eye tail length \\
\hline & enL-exL & Left palpebral fissure length & Ratio & psR-piR/enR-exR & Eye ratio of width to height \\
\hline & & & & $(e n R-e x R+e n L-e x L) / z y R-z y L$ & Length ratio of eyes to face \\
\hline Nose & (11 traits) & & & & \\
\hline Width $^{a}$ & sbalR-sball & Subnasal width & Angle ${ }^{c}$ & (A) prn-n & Nasal bridge angle \\
\hline \multirow[t]{4}{*}{ Height $^{b}$} & $(V) n-s n$ & Frontal nasal height & & (A) prn-sn & Nasolabial angle \\
\hline & $n-s n$ & Profile nasal length & & (A) n-prn-sn & Profile nasal angle \\
\hline & (V) n-prn & Nasal bridge height & & & \\
\hline & (V) prn-sn & Nasal tip height & & & \\
\hline \multirow[t]{2}{*}{ Depth } & $(H)$ n-prn & Nasal bridge depth & & & \\
\hline & $(H)$ prn-sn & Nasal tip protrusion & & & \\
\hline Area & (AR) n-prn-sn & Profile nasal area & & & \\
\hline Mouth & (2 traits) & & & & \\
\hline \multirow[t]{2}{*}{ Height $^{b}$} & (V) cphR-sto & Right upper lip thickness & & & \\
\hline & (V) cphL-sto & Left upper lip thickness & & & \\
\hline Upper eyelid & (30 traits) & & & & \\
\hline \multirow[t]{2}{*}{ Width $^{a}$} & (H) er1-ermax & Right eyelid peak width & Angle ${ }^{c}$ & (A) Tan_er1 7 & Tangent line angle of er $1 \sim$ er 7 \\
\hline & $(H)$ el1-elmax & Left eyelid peak width & & (A) Tan_el1 7 & Tangent line angle of el $1 \sim$ el7 \\
\hline
\end{tabular}


Table 1 Eighty-five facial traits from 23 frontal, 7 profile, and 16 upper eyelid points (Continued)

\begin{tabular}{|c|c|c|c|c|}
\hline \multicolumn{2}{|l|}{ Size-related variables } & \multicolumn{3}{|c|}{ Shape-related variables } \\
\hline Abbreviation & Description & & Abbreviation & Description \\
\hline (H) er1-er7 & Right eyelid width & \multirow[t]{8}{*}{ Ratio } & $(\mathrm{H})$ er1-ermax/(H) er1-er7 & Right eyelid peak position ratio \\
\hline$(\mathrm{H})$ el1-el7 & Left eyelid width & & $(\mathrm{H})$ el1-elmax/(H) el1-el7 & Left eyelid peak position ratio \\
\hline & & & $(V)$ er1-er7/(H) er1-er7 & Right eyelid slant \\
\hline & & & (V) el1-el7/(H) el1-el7 & Left eyelid slant \\
\hline & & & (V) er1-ermax/(H) er1-ermax & Right eyelid medial slant \\
\hline & & & (V) el1-elmax/(H) el1-elmax & Left eyelid medial slant \\
\hline & & & $(\mathrm{V})$ ermax-er7/(H) ermax-er7 & Right eyelid lateral slant \\
\hline & & & (V) elmax- el7/(H) elmax- el7 & Left eyelid lateral slant \\
\hline & & \multirow[t]{4}{*}{ Curvature $^{d}$} & (AC) er1-er7 & Right eyelid average curvature \\
\hline & & & (MC) er1-er7 & Right eyelid maximal curvature \\
\hline & & & (AC) el1-el7 & Left eyelid average curvature \\
\hline & & & (MC) el1-el7 & Left eyelid maximal curvature \\
\hline
\end{tabular}

Trait abbreviations: $c p h$ crista philtri, el left upper eyelid, elmax left upper eyelid peak, en endocanthion, er right upper eyelid, ermax right upper eyelid peak, ex exocanthion, go gonion, $m$ metopion, mtro metopion position on tr-o, $n$ nasion, o ophryon, obi otobasion inferius, obs otobasion superius, pi palpebral inferius, prn pronasale, ps palpebral superius, sbal subalare, sn subnasale, sto stomion, tr trichion, zy zygion, $(A)$ angle, $(A C)$ average curvature, $(A R)$ area, $(H)$ horizontal, $(M C)$ maximal curvature, $(V)$ vertical

${ }^{a}$ Width refers to the horizontal distance between the two landmarks in a frontal (or lateral) image

${ }^{\mathrm{b}}$ Height similarly refers to the vertical distance between the two landmarks in a frontal (or lateral) image

'Angle refers to the angle made from the line through two landmarks and a horizontal line in a frontal (or lateral) image

${ }^{\mathrm{d} C u r v a t u r e ~ i n d i c a t e s ~ t h e ~ r a d i u s ~ o f ~ c i r c l e ~ a t ~ a ~ p o i n t ~} \mathrm{n} 1$ in a frontal upper eyelid

\section{Follow-up study and meta-analysis}

In the following phase, 128 SNPs (17 in frontal, 18 in forehead, 21 in eye, 50 in upper eyelid, and 40 in nose traits) were selected based on the suggestive association $\left(P<5 \times 10^{-6}\right)$ in 65 facial traits, and were validated for their associations in an additional 1926 samples (Phase 2).
Among the 128 variants, 117 SNPs were successfully genotyped and analyzed with related traits (Additional file 8: Table S5). Twenty-one association signals with 11 SNPs (two in face, one in forehead, one in eye, four in upper eyelid, and 13 in nose traits) were replicated with the criterion of $P<0.05$.

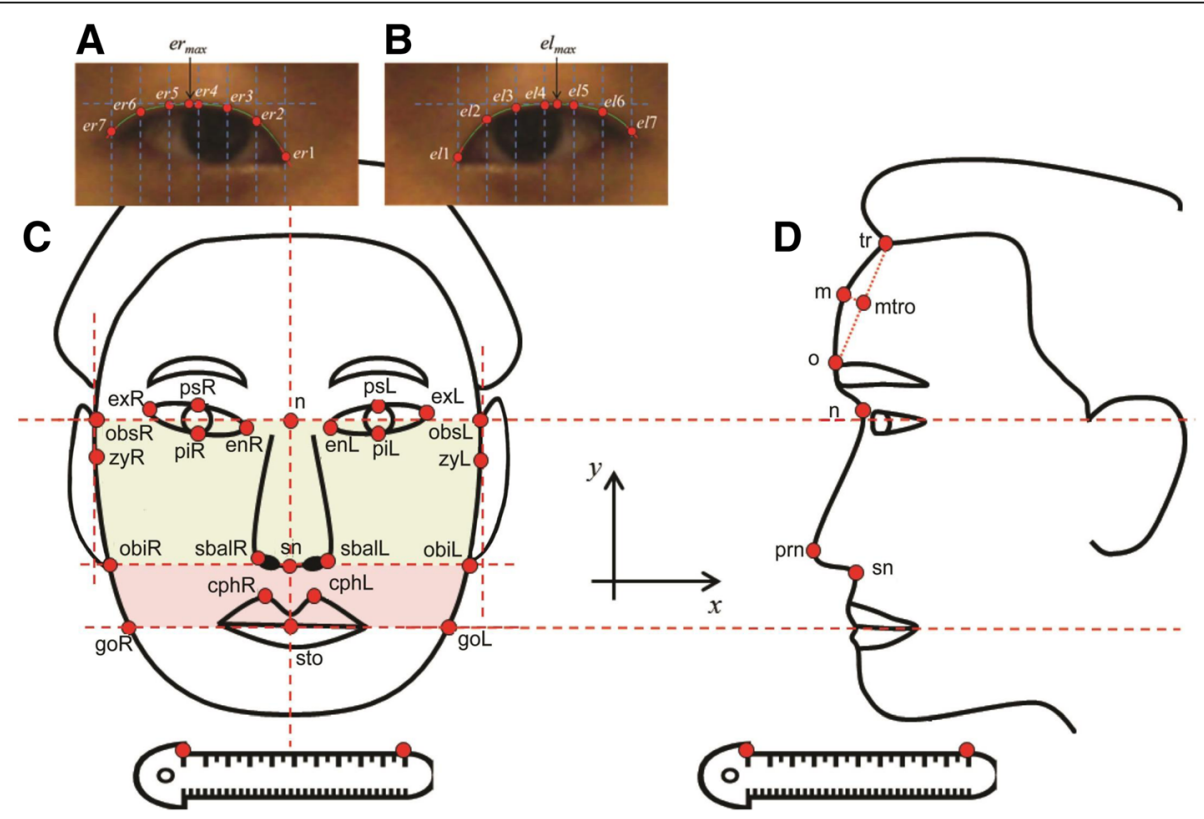

Fig. 1 Facial points used for the facial traits. The participants were photographed in both frontal and profile views, and 23 frontal and seven lateral face points were extracted (modified from Fig. 1 of reference [34]). Facial phenotypes, such as distance, angle, and area, were measured based on in-house facial data acquisition software. a Points in the right eye, $\mathbf{b}$ points in the left eye, $\mathbf{c}$ points and areas in frontal images, $\mathbf{d}$ points in lateral images 
In a meta-analysis of the results of the two cohorts (Phase $1+2$ ), five loci reached genome-wide significance, including rs7567283 (the OSR1-WDR35 locus), rs970797 (the HOXD-MTX2 locus), rs3736712 (the WDR27 locus), rs2193054 (the SOX9 locus), and rs2206437 (the DHX35 locus; Table 2). The genetic regions of these five loci and their association results are depicted as regional association plots in Fig. 2.

The OSR1-WDR35 locus (rs7567283) was associated with the right facial angle of en-ex-go [(A) enR-exR-goR] $\left(P=2.75 \times 10^{-8}\right)$ with regard to the face contour from a frontal view (Fig. 3). The HOXD-MTX2 locus (rs970797) was associated with curvature of the upper eyelid, i.e., the tangent line angle of el3 [(A) Tan_el3] in the left eye $\left(P=7.40 \times 10^{-9}\right)$ and tangent line angle of er3 [(A) Tan_er3] in the right eye $\left(P=3.97 \times 10^{-9}\right)$, which affect eye shape. The WDR27 locus (rs3736712) was associated with eye tail length (psR-exR) $\left(P=8.44 \times 10^{-10}\right)$. The SOX9 locus (rs2193054) exhibited the strongest signal in this study and was associated with nose shape, i.e., profile nasal angle [(A) n-prn-sn $)\left(P=6.17 \times 10^{-17}\right)$ and nasal tip protrusion $[(\mathrm{H})$ prn-sn $]\left(P=5.34 \times 10^{-9}\right)$. The DHX35 locus (rs2206437) was associated with the subnasal width (sbalR-sbalL) $\left(P=1.61 \times 10^{-9}\right)$.

We also analyzed the phenotypic variances explained by $\mathrm{SNP}(\mathrm{s})$ (\%) is obtained by $\mathrm{R}^{2}$ fraction of the associated $\mathrm{SNP}(\mathrm{s})$ from linear regression model $\left(\mathrm{R}^{2}\right.$ of $\mathrm{SNP}(\mathrm{s})$ - $\mathrm{R}^{2}$ of covariates) (Additional file 9: Table S6). As a result, the phenotypic variances explained by the associated SNPs were lower than 1\% in all SNPs.

The loci identified in this study showed multiple associations among the facial traits analyzed. Table 2 shows only the traits that met the genome-wide significance level according to the $P$-value. If the criteria were less stringently applied (i.e., $P<1 \times 10^{-4}$ ), the number of suggestive associated traits increased in the discovery GWAS (Additional file 10: Table S7 and Additional file 11: Figure S4). Most of the suggestive associations showed similar facial traits to those of the five novel variants identified in this study.

\section{Multiple signals in the SOX9 locus}

The SOX9 locus displayed multiple signals, as shown in regional association plots of the discovery GWAS (Fig. 4). These four signals were present in a similarly associated pattern for the five nose traits of nasal tip protrusion $[(\mathrm{H})$ prn-sn], nasal bridge depth $[(\mathrm{H}) \mathrm{n}$-prn], profile nasal area [(AR) n-prn-sn], nasolabial angle [(A) prn-sn], and profile nasal angle [(A) n-prn-sn]. The SNPs representing the signals were rs2193054, rs9910003, rs1859979, and rs9915190, which were located approximately 91, 238, 688 , and $974 \mathrm{~kb}$ upstream of the SOX9 transcription initiation site, respectively. Both rs2193054 (91 kb) and rs1859979 $(688 \mathrm{~kb})$ met the genome-wide significance level in the discovery GWAS, whereas the two SNPs rs9910003 (238 kb) and rs9915190 (974 kb) showed rather weak associations.

These four variants were tested for their independence of association signals by investigating the pair-wise linkage disequilibrium (LD) and conducting conditional association analyses. The four SOX9 SNPs showed no LD in both the Phase 1 and 1000 Genomes populations, except for weak LD detected between rs2193054 and rs9910003 (0.19 in the Phase 1 population and 0.08-0.25 in the 1000 Genomes database) (Additional file 12: Table S8). The association of rs9910003 did not persist after conditioning with rs2193054, although the associations of other variants persisted (Additional file 13: Table S9). Therefore, these SNPs (rs2193054, rs1859979, and rs9915190) were further genotyped with the 1940 Phase 2 samples for validation of their associations (Fig. 4, Table 3). These three SNPs passed the threshold of genome-wide significance after meta-analysis, and further examination of these variants with HaploReg [19] identified the promoter and enhancer histone marks, DNase hypersensitivity regions, and changed motifs in their sequences (Additional file 14: Table S10).

\section{Replication analysis of previously reported facial trait GWAS loci}

We performed a replication analysis for previously reported face morphology GWAS loci [12-17] (Table 4). A total of 41 lead SNPs were selected for this analysis. Since the Affymetrix Genome-Wide Human SNP array 5.0 genotype platform did not include these SNPs, except for rs6555969 (C5orf50), we extracted proxy SNPs of these 41 lead SNPs using LDlink (https://analysis tools.nci.nih.gov/LDlink) [20] under the criterion of $\mathrm{r}^{2}>$ 0.6. After filtering low-frequency SNPs [minor allele frequency (MAF) < 0.05], X chromosome SNPs, and GWAS QC-failed SNPs, 63 proxy SNPs from 13 lead SNPs were selected and examined for their association with relevant facial traits examined in this study. As shown in Table 4, one proxy SNP per lead SNP that showed the highest LD $\left(\mathrm{r}^{2}\right)$ value among the proxy SNPs was identified.

The rs4648379 locus was previously associated with nose width and nose height [13], and its proxy SNP rs4648478 $\left(\mathrm{r}^{2}=0.61\right)$ showed an association of $P=$ $5.70 \times 10^{-6}$ with profile nasal angle [(A) n-prn-sn] in our analysis. Because there were 11 nose-related traits in this study, the criterion of replication significance was set to $P=4.5 \times 10^{-3}$ considering the Bonferroni multiple correction. Therefore, the SNP rs4648379 appeared to be replicated in this study. Similarly, the locus rs2045323 was reported to be associated with three nose phenotypes (columella inclination, nose protrusion, and nose tip angle) [14], and this locus was replicated in this study, demonstrating an association with subnasal width 


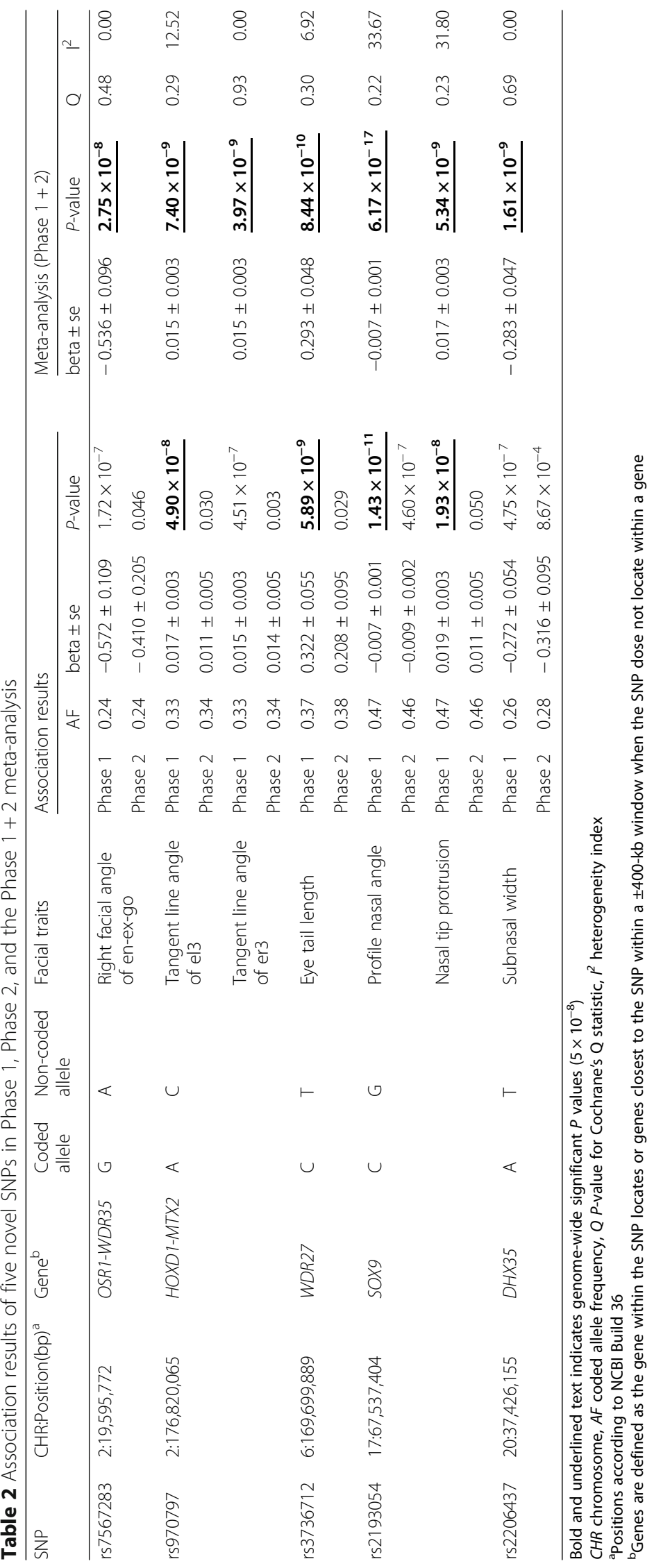



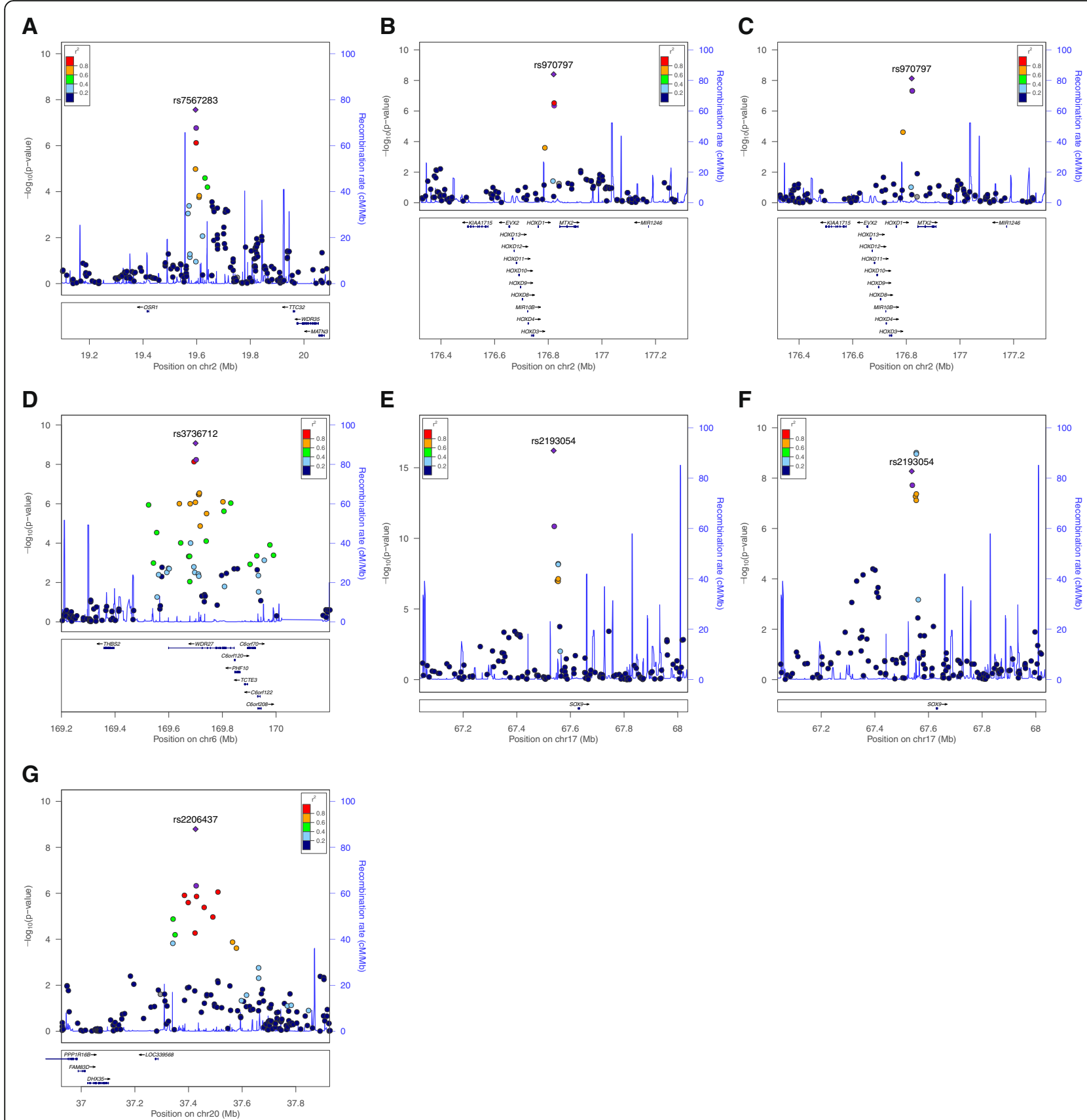

Fig. 2 Regional association plots for five novel loci across a 1-Mb window. Association of individual SNPs in the discovery GWAS plotted as -log 10 (P) against the chromosomal base-pair position. The $y$-axis on the right shows the recombination rate, estimated from the HapMap CHB and JPT populations. P-values are from the discovery phase. The purple circle and diamond represent the results of discovery and meta-analysis (Phase $1+2$ ), respectively. Seven signal plots for five novel SNPs are illustrated, which show the genome-wide significant $P$-values in the meta-analysis (Phase 1+2). a rs7567283 of the right facial angle of en-ex-go [(A) enR-exR-goR], b rs970797 of the tangent line angle of er3 [(A) Tan_er3], c rs970797 of the tangent line angle of el3 [(A) Tan_el3], d rs3736712 of eye tail length (psR-exR), e rs2193054 of profile nasal angle [(A) n-prn-sn], $\mathbf{f}$ rs2193054 of nasal tip protrusion [(H) prn-sn], and $\mathbf{g}$ rs2206437 of subnasal width (sbalR-sbalL)

(sbalR-sbalL) (proxy SNP rs4315762, $\mathrm{r}^{2}=0.68, P=4.84 \times$ $10^{-6}$ ). Another SNP, rs1852985, associated with nose bridge breadth [14], was also replicated with nasal bridge angle [(A) prn-n] (rs1284964, $\left.\mathrm{r}^{2}=0.85, P=2.28 \times 10^{-5}\right)$, and rs2424399, previously associated with nasal width
[15], was replicated with subnasal width (sbalR-sbalL) (rs6082475, $\left.\mathrm{r}^{2}=1.0, P=7.28 \times 10^{-4}\right)$.

In addition, we performed another replication analysis of the SNPs identified by Pickrell et al. [21], who used self-reported information in a very large cohort 


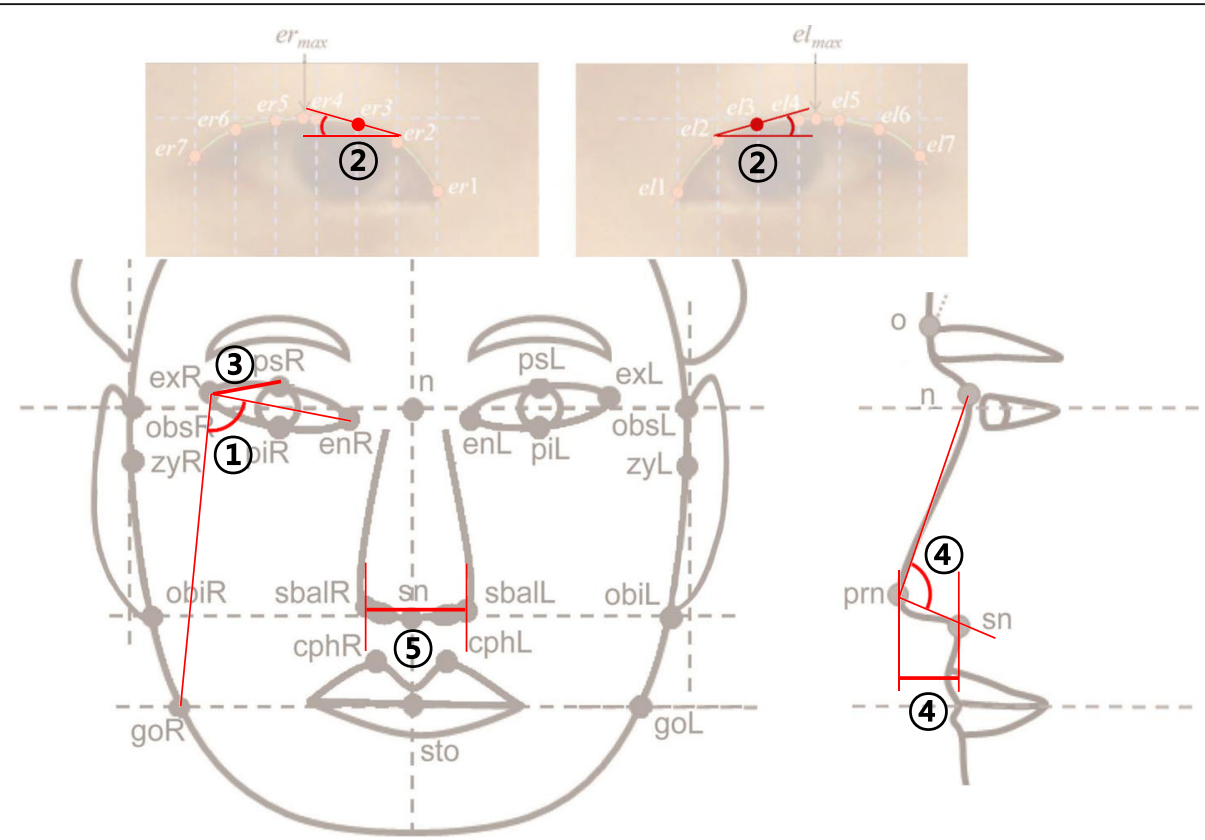

Fig. 3 Associated facial traits for five novel SNPs. Five novel SNPs and the associated facial traits are illustrated on the frontal, lateral, and eye images. (1) rs7567283 (OSR1-WDR35) and right facial angle, (2) rs970797 (HOXD1-MTX2) and left and right curvature of the upper eyelid, (3) rs3736712 (WDR27) and eye tail length, (4) rs2193054 (SOX9) and nose shape (angle and height), and (5) rs2206437 (DHX35) and subnasal width

$(n>70,000)$. They identified 23 lead SNPs associated with nose size. After applying the same filtering process described above, eight lead SNPs were further selected for analysis in our sample, and their 40 proxy SNPs were examined for association with nose traits. As a result, two SNPs, rs767764 and rs6101567, were replicated in the present study (Table 4).

\section{Discussion}

Through the first two-stage GWAS on facial morphology in the Korean population, we identified five genetic loci that were significantly associated with facial traits, including facial frontal contour, eye shape, and nose shape. Only one SNP, rs97097, in the HOXD-MTX2 locus was found in the GWAS catalogue and the UK Biobank, which has been associated with monobrow and earlobe attachment $[15,22]$. In addition, during the review process of this paper, Claes et al. reported the facial trait association of two SNPs in HOXD1-MTX2 and SOX9 (rs970797 and rs5821892, respectively) [23].

\section{Face morphology GWAS identified genes for craniofacial malformations}

Among the associations, two loci seemed to be related to known craniofacial malformations. The SNP rs2193054 in the SOX9 locus is located at the proximal break point cluster region of campomelic dysplasia (CMPD [MIM 114290]). CMPD is caused by mutations in the SOX9 gene at $17 \mathrm{q} 24.3$ and mainly manifests as skeletal defects such as tubular bone bowing by autosomal dominant inheritance (Additional file 15: Table S11) [24, 25]. CMPD frequently presents as abnormal facial features, including a flat face and depressed nasal bridge, suggesting an important role for SOX9 in craniofacial chondrocyte differentiation.

Another variant, rs7567283 in $2 \mathrm{p} 24.1$, was located $378 \mathrm{~kb}$ downstream of the WDR35 gene, which belongs to the WD repeat family, providing a beta propeller scaffold for the assembly of multiple protein complexes [26]. The WDR35 gene is related to cranioectodermal dysplasia 2 (CED2 [MIM 613610]), which manifests as forehead bossing, dolichocephaly, and metaphyseal dysplasis [27]. Dolichocephaly is a condition in which the head is longer than expected relative to its width and is correlated with frontal face width traits associated with the variant rs7567283 in the WDR35 locus (Additional file 15: Table S11).

\section{Allele frequencies of associated variants are different among ethnic groups}

Interestingly, four out of five associated variants showed differences in allele frequencies among ethnic groups, suggesting that these variants may contribute to facial differences among ethnic groups (Additional file 16: Table S12). The reference allele $G$ in rs7567283 (OSR1-WDR35) resulted in a narrower frontal face than that of the alternate allele (A). Based on the 1000 Genome Project data, the $\mathrm{G}$ allele is less frequent in East 


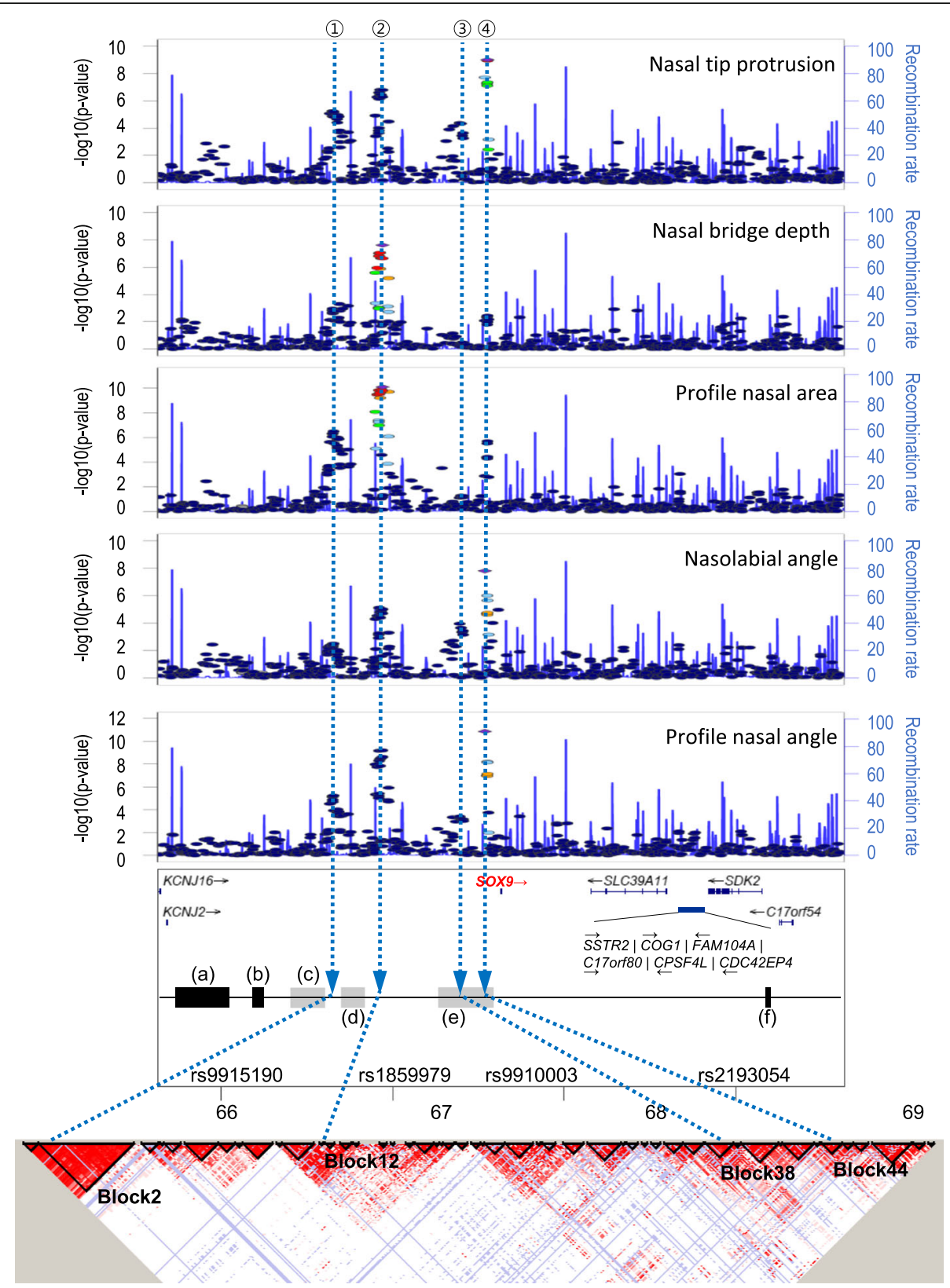

Fig. 4 Association signals in the SOX9 locus and genomic environment surrounding SOX9 across a 4-Mb window. The upper five panels present multiple signals, (1) rs9915190, (2) rs1859979, (3) rs9910003, and (4) rs2193054, in the SOX9 locus for five nose traits: nasal tip protrusion [(H) prn-sn], nasal bridge depth [(H) n-prn], profile nasal area [(AR) n-prn-sn], nasolabial angle [(A) prn-sn], and profile nasal angle [(A) n-prn-sn]. These are plotted as $-\log _{10}(P)$ against base-pair position on chromosome $17(\mathrm{Mb})$ and all $P$-values are from the discovery phase. The sixth panel shows genes and regulatory domains. Gray boxes represent approximate boundaries of translocation breakpoint clusters, and black boxes represent microdeletions. (a) Sp4 [31], (b) F1 [31], (c) Pierre Robin sequence (PRS) breakpoint cluster [31, 32], (d) distal breakpoint cluster [30], (e) proximal breakpoint cluster [30], and (f) Sp2 [31]. The last panel presents LD blocks based on the HapMap database (HapMap Phase II JPT + CHB, hg18)

Asians (24\%) than in both Europeans (82\%) and Africans (67\%). Similarly, the reference allele $C$ in rs3736712 (WDR27) results in longer eye tail lengths than alternate alleles and is less frequent in East Asians (39\%) than in both Europeans (93\%) and Africans (93\%).

The reference allele C of rs2193054 (SOX9) results in higher nasal protrusion than the alternate allele and is less frequent in Africans (24\%) than in both East Asians (45\%) and Europeans (50\%). The profile nose size-increasing alleles of the three other conditionally independent SOX9 variants (rs9915190, rs1859979, and rs9910003) are less frequent in East Asians that in both Europeans and Africans (54, 44, and 30\% in East Asians versus 64, 95, and 46\% in Europeans and 83, 90, and 


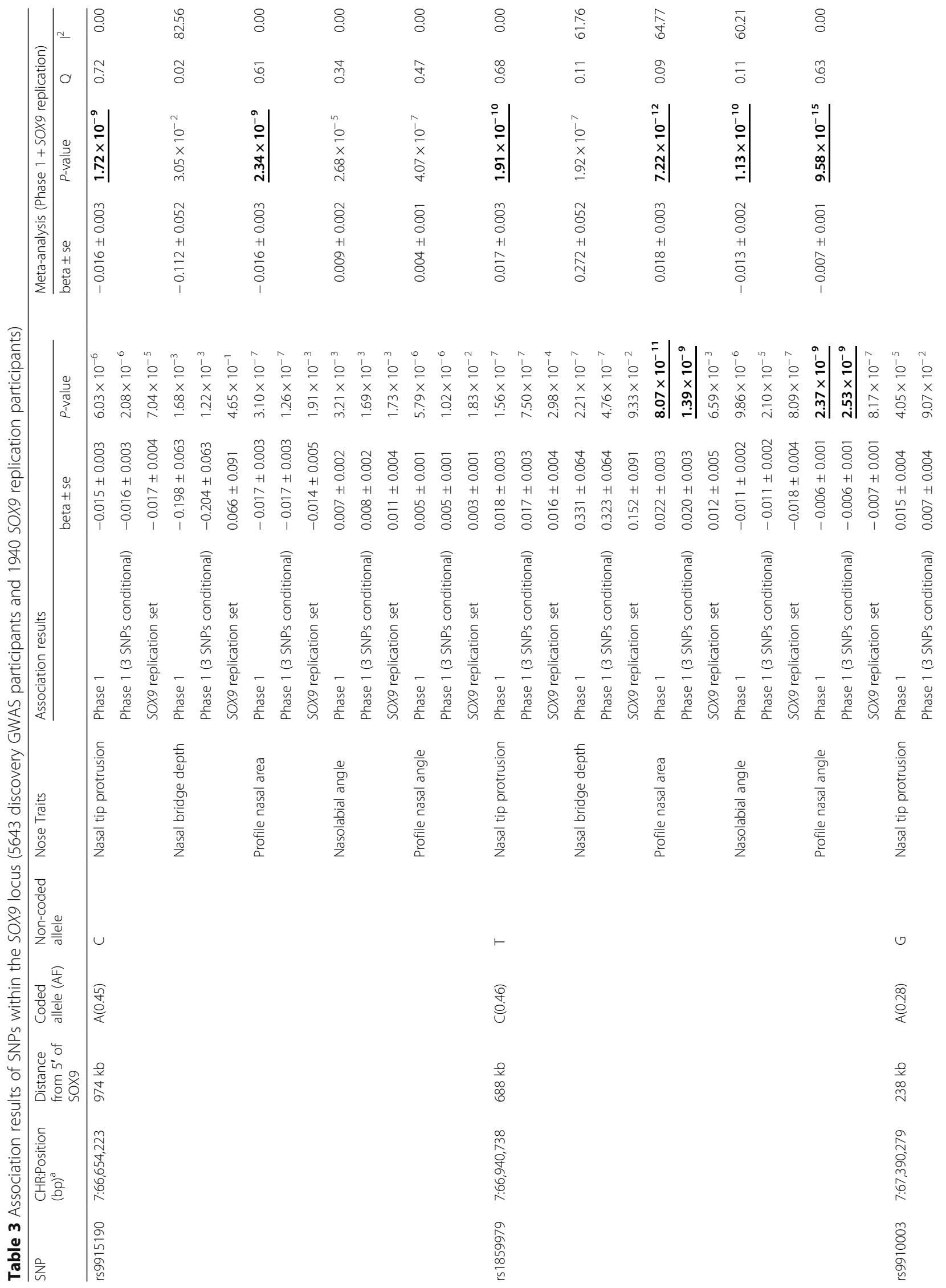




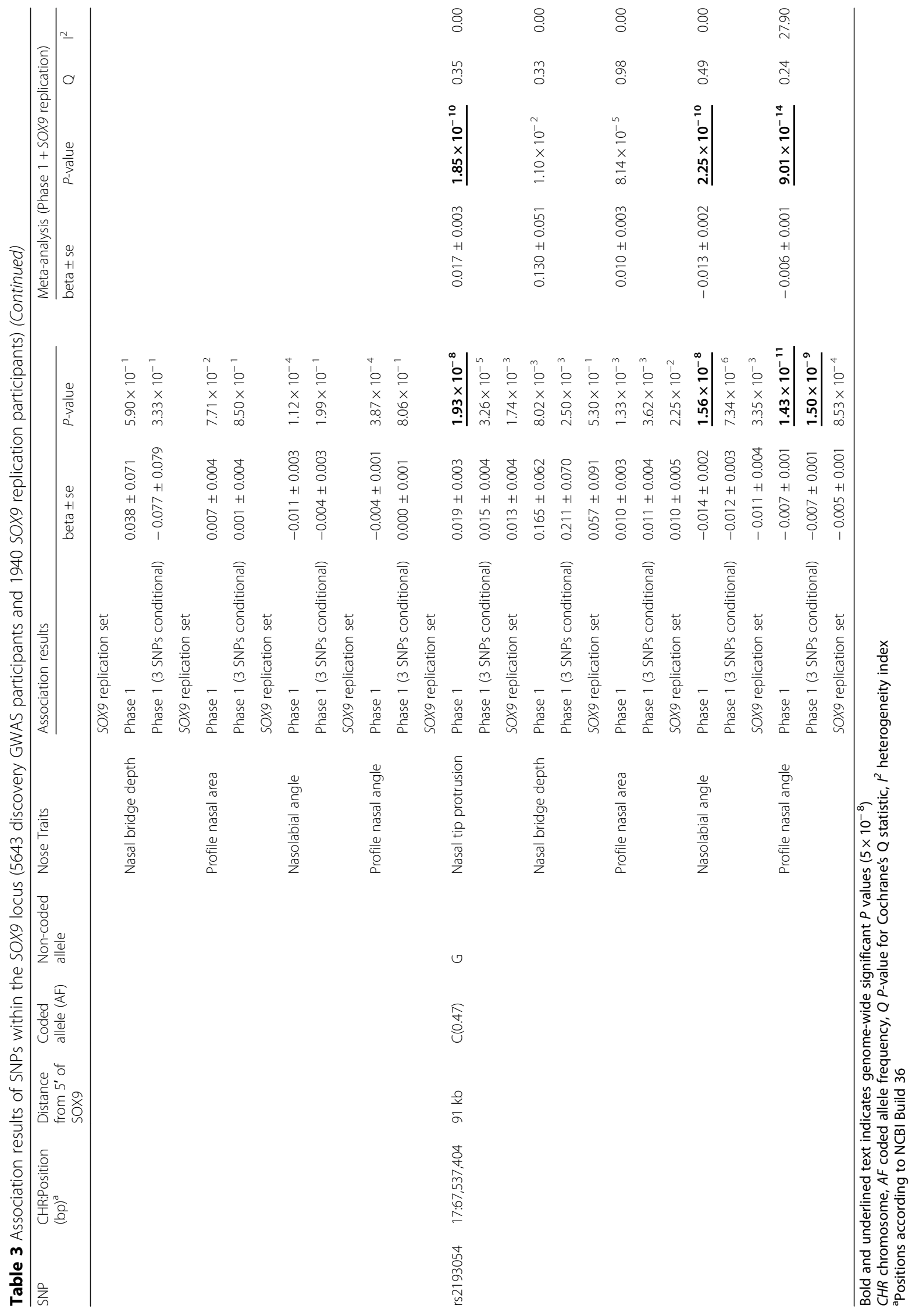




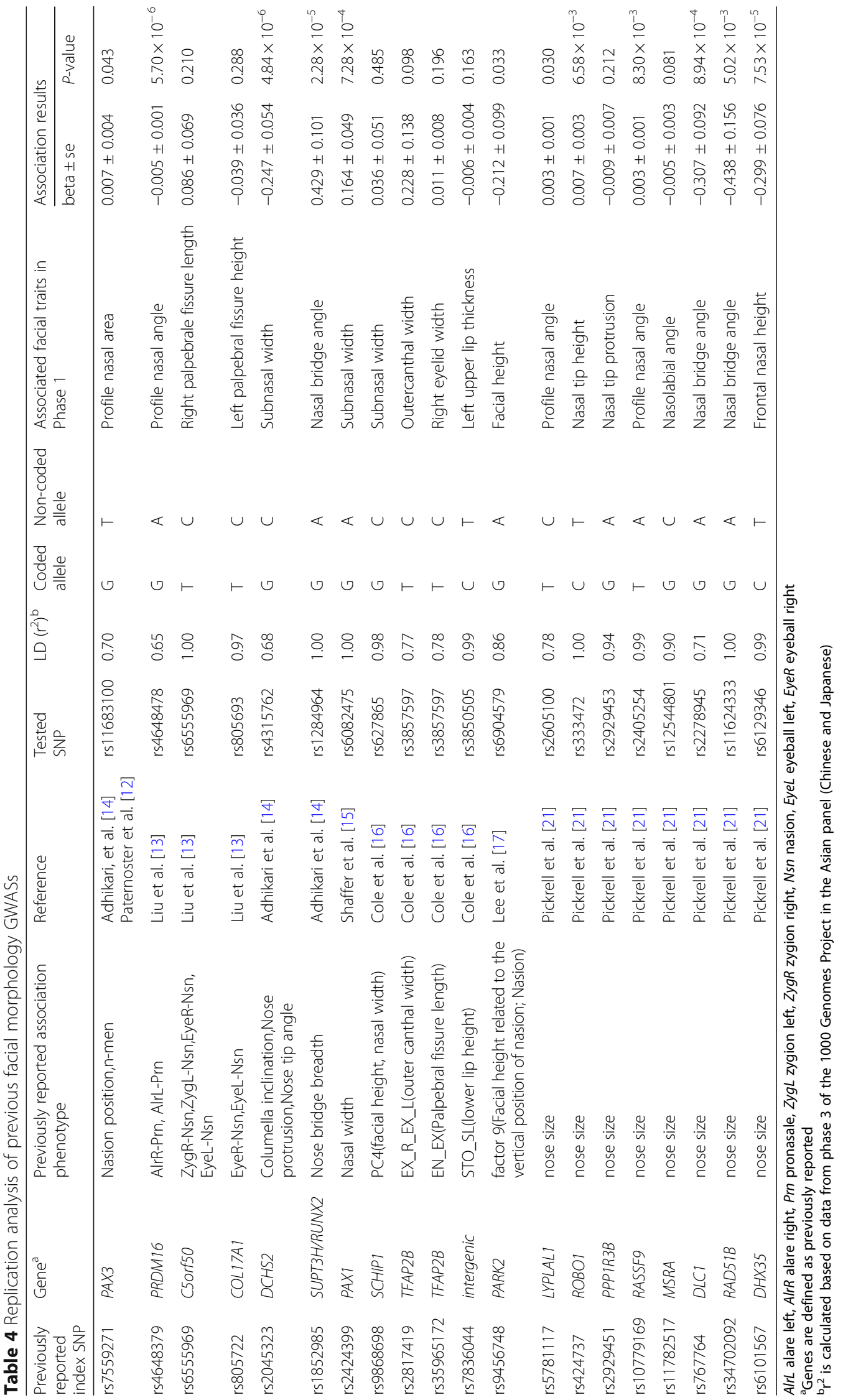


$57 \%$ in Africans, respectively). The T allele of rs2206437 (DHX35) results in a wider and lower nose than the alternate allele A from the front view and is more frequent in both East Asians (77\%) and Africans (75\%) than in Europeans (52\%). Thus, we found several significant variants for nose shape manifesting differential allele frequencies among different ethnic populations. Indeed, several previous GWASs have identified variants associated with nose traits (Table 4), suggesting that nasal traits are highly heritable in humans [28].

\section{Multiple association signals in the SOX9 locus confirmed the long-range regulation of the SOX9 gene}

Three association signals of rs2193054 (91 kb), rs1859979 (688 kb), and rs9915190 (974 kb) upstream of the SOX9 gene were identified as independently associated based on both the LD block and conditional analyses. Consistent with these findings, genetic analyses of patients with CMPD and Pierre Robin sequence (PRS, [MIM 261800]) revealed multiple translocation breakpoints and deletions in the $\sim 3-\mathrm{Mb}$ region spanning the SOX9 coding region, indicating that $S O X 9$ may be modulated by multiple regulatory sequences within the long-distance range [29]. The proximal breakpoint cluster [30] [50-375 kb, depicted as (e) in the lower gene map panel of Fig. 4], distal breakpoint cluster [30] [789-932 kb, Fig.4 (d)], and PRS breakpoint cluster $[31,32][\sim 1.13 \mathrm{Mb}$, Fig. 4 (c) $]$ are translocation breakpoints upstream of the SOX9 gene, and deletions were identified throughout the region over $1 \mathrm{Mb}$ upstream of SOX9 [Fig. 4 (a) and (b)] and in the downstream region [Fig. 4 (f)] [31, 33].

To gain more insights into the regulatory functions of the associated variants, we aligned the SNPs with mutation sites found in patients with CMPD and PRS. The location of rs2193054 closest to the SOX9 gene corresponding to the proximal breakpoint cluster region rs1859979 was just downstream of the distal breakpoint cluster, and rs9915190 was located between the PRS and the distal breakpoint cluster. Therefore, patients with CMPD harboring a chromosomal break in the proximal breakpoint cluster may lose both regulatory elements at $688 \mathrm{~kb}$ (rs1859979, (2) in the lower gene map panel of Fig. 4) and 974 kb (rs9915190, (1) in Fig. 4), whereas patients with CMPD harboring a break in the distal breakpoint cluster may lose only the element at $974 \mathrm{~kb}$ upstream of the $\mathrm{SOX} 9$ gene.

\section{Study limitations}

The main limitation of the study was that the facial measurements were derived from two-dimensional images rather than three-dimensional images, which could be the gold standard in the GWAS of facial traits. Given this limitation, we tried to measure more facial traits than evaluated in previous studies, such as the angles, ratios, and curvatures, as well as conventional distances including widths and heights.

\section{Conclusion}

We identified five novel face morphology loci that were associated with facial frontal contour, nose shape, and eye shape. Our findings were further emphasized by three observations: 1) two of the loci have been implicated in craniofacial malformations; 2) the allele frequencies of four variants differed among ethnic groups; and 3) the SOX9 locus contained three independent association signals. Thus, face morphology GWASs may expand our understanding of craniofacial malformations and provide useful genetic information for externally visible characteristics in forensic science.

\section{Methods}

\section{Study participants}

The participants for the discovery GWAS (Phase 1) were recruited from two regions in South Korea (Ansan and Ansung) from 2009 to 2012 for the Korean Genome and Epidemiology Study (KoGES) [34]. The criterion for inclusion of participants in the study was the availability of facial images and genome-wide genotype data. The criteria for exclusion were as follows: a history of cancer, gender inconsistencies, cryptic relatedness, low genotype call rate $(<95 \%)$, and sample contamination, as previously described [34]. A total of 5643 individuals (2648 men and 2995 women) met the requirements and were selected for the discovery GWAS (Phase 1).

The follow-up analysis (Phase 2) was performed using 2009 individuals from 19 Oriental medical clinics recruited from 2007 to 2012 for the Korea Constitution Multicenter Study (KCMS). Among the 2009 study participants, we extracted 1926 samples (687 men and 1239 women) who were over the age of 20 and showed high genotype call rates ( $\geq 95 \%$ ).

To validate the multiple association signals in the SOX9 locus, we used another independent replication set of participants recruited from 2011 to 2012 for the Korea Constitution Multicenter Study (KCMS). After applying the above-mentioned inclusion and exclusion criteria, 1940 individuals (587 men and 1353 women) were used to confirm the association between nose traits and multiple variants within the SOX9 locus.

\section{Facial traits}

The overall procedure for feature extraction is shown in Additional file 17: Figure S5. The participants were confirmed to not be wearing any cosmetic make-up and were photographed from both frontal and lateral views using a digital camera (DSLR Nikon D90 with a Nikon AF 50 -mm F1.8D lens, $3216 \times 2136$ pixels) under the following standard conditions: the hair was pulled back 
with a hair band; the center points of the two pupils and the two points connecting the facial contour and upper auricular perimeters (e.g., points obsR and obsL from the frontal image shown in Fig. 1) were on the same horizontal line; and a ruler was placed approximately $10 \mathrm{~mm}$ below the chin to convert pixels into millimeters [35].

Facial feature points in frontal and lateral images were automatically extracted by detecting and analyzing the face, eyes, nose, mouth, and contours via our own developed program in Visual Studio $\mathrm{C}++$ with the use of OpenCV (Open Source Computer Vision Library). Given an input image, the region of interest (ROI) was reduced in stages by detecting the face, eyes, nose, and mouth sequentially with Adaboost-based detectors $[36,37]$. In each ROI, the facial feature points were found from facial contours obtained through histogram-based image segmentation. The positions of the extracted points were confirmed by a well-trained operator, and the same extraction procedure for facial feature points was applied to both the discovery and replication sets. The accuracy of the automatic landmarking was $98.8 \%$ on average, and we excluded the data for cases with inaccurate landmarking.

The facial traits analyzed in the study were delineated by distance, distance ratio, angle, area, and eye curvature from 23 frontal and seven lateral facial points (Fig. 1 and Table 1). The detailed descriptions of these traits were presented in previous studies $[38,39]$. For linear regression analysis, 15 severely skewed facial traits, including upper forehead slant depth $[(\mathrm{H})$ tr-m], brow ridge protrusion $[(\mathrm{H}) \mathrm{o}-\mathrm{n}]$, metopion eminence depth (m-mtro), left eyelid average curvature [(AC) el1-el7], left eyelid maximal curvature [(MC) el1-el7], right eyelid average curvature [(AC) el1-el7], right eyelid maximal curvature [(MC) er1-er7], profile nasal length ( $n-s n)$, nasal tip height $[(\mathrm{V})$ prn-sn], nasal tip protrusion $[(\mathrm{H})$ prn-sn], profile nasal area [(AR) n-prn-sn], nasolabial angle [(A) prn-sn], profile nasal angle [(A) n-prn-sn], right upper lip thickness $[(\mathrm{V}) \mathrm{cphR}$-sto], and left upper lip thickness [(V) cphL-sto], were $\ln$-transformed. We removed outliers in each facial trait, which were defined using the first and third quartiles and interquartile range of each facial trait. In each facial trait, outliers under the first quartile $-2.0 \times$ interquartile range or over the third quartile $+2.0 \times$ interquartile range were excluded.

\section{Genotyping}

Genotyping of DNA from the discovery GWAS (Phase 1) population was performed using an Affymetrix GenomeWide Human SNP array 5.0 (Affymetrix, Santa Clara, CA, USA), as described in a previous report [34]. Among 500,568 SNPs in the Affymetrix SNP array, 311,944 autosomal SNPs were examined in the GWAS, after excluding SNPs with a high missing call rate $(>5 \%)$, low
MAF $(<0.05)$, and deviation from Hardy-Weinberg equilibrium $(P<0.0001)$.

For the follow-up of SNPs that met the suggestive threshold of association with facial traits $\left(P<5 \times 10^{-6}\right)$, we conducted LD pruning and selected the 128 sentinel SNPs that tagged each locus. Replication genotyping of the 1926 samples (Phase 2) was performed using an Applied Biosystems QuantStudio 12 K Flex Real-Time PCR System. Genotyping quality was controlled by excluding SNPs with low call rates $(<95 \%)$, low MAF $(<0.01)$, and bad genotype clustering. Individuals with a high missing genotype call rate (>5\%) were excluded from analysis.

The genotypes of three variants (rs9915190, rs1859979, and rs2193054) within the SOX9 locus were determined using an unlabeled oligonucleotide probe (UOP) on a polymorphic nucleotide for 1940 participants (SOX9 replication set). The detailed process of genotyping using a UOP for the variant was described in a previous report $[40,41]$. An aliquot of the polymerase chain reaction amplicon including the SNP site was diluted in a solution containing $1 \mathrm{mM}$ UOP, $5 \mathrm{mM}$ SYTO-9 (Invitrogen, Carlsbad, CA, USA), $12.5 \mathrm{mM}$ EDTA, and $10 \mathrm{mM}$ Tris ( $\mathrm{pH} 8.0)$. The DNA in the UOP sample sequentially underwent denaturation $\left(95^{\circ} \mathrm{C}\right.$ for $\left.5 \mathrm{~s}\right)$, annealing $\left(60{ }^{\circ} \mathrm{C}\right.$ for $1 \mathrm{~min}$ ), and melting with a gradual increase to $74{ }^{\circ} \mathrm{C}$ at a rate of $1{ }^{\circ} \mathrm{C} / \mathrm{s}$; the fluorescence emission was read using a Light Cycler 480 instrument (Roche, Indianapolis, IN, USA). The genotype was determined from three melting patterns of the UOP (major homozygote, heterozygote, and minor homozygote).

\section{Statistical analysis}

GWASs were performed for discovering variants associated with facial traits such as frontal and lateral images of the eye, nose, contour, and other features, using PLINK version 1.09 [42] by linear regression analysis in an additive model, with adjustment for age, sex, and BMI. The cut-off $P$-value for discovery GWAS (Phase 1) was $5.0 \times 10^{-6}$. Quantile-quantile plots for facial traits were constructed with the distribution of observed $P$-values against the theoretical distribution of expected $P$-values. A regional association plot for a genomic region of $1 \mathrm{Mb}$ centered on the peak SNP was constructed using LocusZoom [43].

In the follow-up analysis (Phase 2), multiple linear regression analysis was performed to determine the association of 117 SNPs in 1926 participants with the corresponding facial traits, with adjustment for age, sex, and BMI, using PLINK v1.09.

Conditional analyses were performed to identify the $\mathrm{SNP}(\mathrm{s})$ in the SOX9 locus that were independently associated with nose traits in Phase 1 subjects. Multiple linear regression analysis of each SOX9 SNP was carried out for each nose trait, with adjustment for age, sex, 
BMI, and another SOX9 SNP (or the three other SOX9 SNPs together). In addition, we carried out multiple linear regression analysis using $\mathrm{R}$ version 3.0 .2 to validate multiple association signals for nose traits in another independent replication set including 1940 participants (SOX9 replication set).

All meta-analysis calculations were implemented in PLINK and METAL [44] under the assumption of fixed effects using Cochran's Q test to determine between-study heterogeneity. The SNPs in the combined analysis were considered significant when $P$-values were below $5.0 \times$ $10^{-8}$ (as the traditional genome-wide significance level).

For eQTL analysis, databases from GTExPortal [45] and BRAINEAC [46] were used, along with additional data from Schadt et al. [47], Westra et al. [48], and Fairfax et al. [49]. Functional annotations such as chromatin structure, methylation, protein motifs, and transcription factor binding were summarized using HaploReg [19], and functional variant scores were calculated using RegulomeDB [50].

\section{Additional files}

Additional file 1: Table S1. Correlation between face traits and covariates. (XLSX $18 \mathrm{~kb}$ )

Additional file 2: Table S2. Correlations of face traits. (XLSX $88 \mathrm{~kb}$ )

Additional file 3: Table S3. Heritability of 85 facial traits from the Phase 1 population. (DOCX $19 \mathrm{~kb})$

Additional file 4: Figure S1. Study design for facial morphology GWASs. (PDF $116 \mathrm{~kb}$ )

Additional file 5: Table S4. Characteristics of the study participants. (DOCX $32 \mathrm{~kb}$ )

Additional file 6: Figure S2. Q-Q plots for the discovery GWAS (85 facial traits). (PDF $1945 \mathrm{~kb}$ )

Additional file 7: Figure S3. Manhattan plots for the discovery GWAS (85 facial traits). (PDF $2645 \mathrm{~kb}$ )

Additional file 8: Table S5. Association results in Phase 1, Phase 2, and the Phase $1+2$ meta-analysis (63 phenotypes, 117 SNPs). (DOCX 78 kb)

Additional file 9: Table S6. Phenotypic variance explained by five faceassociated SNPs shown in Table 2 in the Phase 1 population (DOCX 15 kb)

Additional file 10: Table S7. Additional associated facial traits of five novel SNPs in the discovery GWAS $(P<0.0001)$. (DOCX $18 \mathrm{~kb}$ )

Additional file 11: Figure S4. Multiple associations of five loci among the 85 facial traits in the discovery GWAS. (PDF $266 \mathrm{~kb}$ )

Additional file 12: Table S8. Pairwise linkage disequilibrium analyses with four nose-associated SNPs in the SOX9 locus. (DOCX $16 \mathrm{~kb}$ )

Additional file 13: Table S9. Results of conditional analysis for four variants in the upstream region of SOX9 (Phase 1). (DOCX $34 \mathrm{~kb}$ )

Additional file 14: Table S10. Functional analysis of three variants in the upstream region of SOX9. (DOCX $15 \mathrm{~kb}$ )

Additional file 15: Table S11. Comparison of clinical phenotypes and facial traits. (DOCX $14 \mathrm{~kb}$ )

Additional file 16: Table S12. Comparisons of allele frequencies between Koreans and other populations from the 1000 Genomes Project Phase 3. (DOCX $16 \mathrm{~kb}$ )

Additional file 17: Figure S5. The procedure for facial feature point extraction. (PDF $362 \mathrm{~kb}$ )

\section{Abbreviations}

BMl: Body mass index; CED2: Cranioectodermal dysplasia 2; CMPD: Campomelic dysplasia; GWAS: Genome-wide association study; KCMS: Korea Constitution Multicenter Study; KoGES: Korean Genome and Epidemiology Study; LD: Linkage disequilibrium; MAF: Minor allele frequency; OpenCV: Open Source Computer Vision Library; PRS: Pierre Robin sequence; ROI: Region of interest; SNP: Single nucleotide polymorphism; UOP: Unlabeled oligonucleotide probe

\section{Acknowledgements}

This study involved bioresources provided by the National Biobank of Korea, the Centers for Disease Control and Prevention, Republic of Korea (4845-301, 4851-302, and 4851-307). We thank the TheragenEtex Bio Institute for providing genotyping services.

\section{Funding}

This research was supported by the Bio\&Medical Technology Development Program of the National Research Foundation funded by the Ministry of Science, ICT \& Future Planning (grant nos. 2006-2005173 and 2006-2005175), by the research program of Korea Institute of Oriental Medicine (No. K18092), and by the Forensic Science Research Project 2014 of the Supreme Prosecutors' Office, Republic of Korea. The funding bodies did not contribute to the design of the study, sample collection, analysis, interpretation of data, or in writing the manuscript.

Availability of data and materials

All data generated or analyzed during this study are included in this published article and its supplementary information files.

\section{Authors' contributions}

SC, JEL, JYK, and BO designed this face morphology GWAS project. JHD and SWL carried out facial traits measurements and CS and NHC collected GWAS samples. SC, JEL, and AYP carried out association analyses and the meta-analysis. JMN analyzed multiple signals in the SOX9 locus. SC, JEL, JSK, KMW, SHL, and BO analyzed and interpreted the data. SC, JEL, JOK, and BO wrote the manuscript. All authors read and approved the final manuscript.

Ethics approval and consent to participate

All participants provided written informed consent to participate in the study. The study was approved by the Institutional Review Board of the Korea National Institute of Health and the Korea Institute of Oriental Medicine.

Consent for publication

Not applicable

Competing interests

The authors declare that they have no competing interests.

\section{Publisher's Note}

Springer Nature remains neutral with regard to jurisdictional claims in published maps and institutional affiliations.

\section{Author details}

${ }^{1}$ Future Medicine Division, Korea Institute of Oriental Medicine, Daejeon 34054, Republic of Korea. ${ }^{2}$ Department of Biochemistry and Molecular Biology, School of Medicine, Kyung Hee University, Seoul 02447, Republic of Korea. ${ }^{3}$ Mibyeong Research Center, Korea Institute of Oriental Medicine, Daejeon 34054, Republic of Korea. ${ }^{4}$ Division of Pulmonary Sleep and Critical Care Medicine, Department of Internal Medicine, Korea University Ansan Hospital and Institute of Human Genomic Study, Korea University Ansan Hospital, Ansan 15355, Republic of Korea. ${ }^{5}$ Department of Preventive Medicine, Ajou University School of Medicine, Suwon 16499, Republic of Korea. 'DNA Forensic Division, Supreme Prosecutors' Office, Seoul 06590, Republic of Korea. ${ }^{7}$ KM Fundamental Research Division, Korea Institute of Oriental Medicine, Daejeon 34054, Republic of Korea. 


\section{Received: 28 August 2017 Accepted: 12 June 2018}

Published online: 19 June 2018

\section{References}

1. Brinkley JF, Borromeo C, Clarkson M, Cox TC, Cunningham MJ, Detwiler LT, Heike $\mathrm{CL}$, Hochheiser $\mathrm{H}$, Mejino $\mathrm{J}$, Travillian RS, et al. The ontology of craniofacial development and malformation for translational craniofacial research. Am J Med Genet C Semin Med Genet. 2013;163C(4):232-45.

2. Kapp-Simon KA, Simon DJ, Kristovich S. Self-perception, social skills, adjustment, and inhibition in young adolescents with craniofacial anomalies. Cleft Palate Craniofac J. 1992;29(4):352-6.

3. Macgregor FC. Facial disfigurement: problems and management of social interaction and implications for mental health. Aesthet Plast Surg. 1990; 14(4):249-57.

4. Walsh S, Chaitanya L, Clarisse L, Wirken L, Draus-Barini J, Kovatsi L, Maeda H, Ishikawa T, Sijen T, de Knijff P, et al. Developmental validation of the HirisPlex system: DNA-based eye and hair colour prediction for forensic and anthropological usage. Forensic Sci Int Genet. 2014;9:150-61.

5. Walsh S, Liu F, Wollstein A, Kovatsi L, Ralf A, Kosiniak-Kamysz A, Branicki W, Kayser M. The HlrisPlex system for simultaneous prediction of hair and eye colour from DNA. Forensic Sci Int Genet. 2013;7(1):98-115.

6. Claes P, Liberton DK, Daniels K, Rosana KM, Quillen EE, Pearson LN, McEvoy B, Bauchet M, Zaidi AA, Yao W, et al. Modeling 3D facial shape from DNA. PLoS Genet. 2014:10(3):e1004224.

7. Toma AM, Zhurov Al, Playle R, Marshall D, Rosin PL, Richmond S. The assessment of facial variation in 4747 British school children. Eur J Orthod. 2012;34(6):655-64

8. Johannsdottir B, Thorarinsson F, Thordarson A, Magnusson TE. Heritability of craniofacial characteristics between parents and offspring estimated from lateral cephalograms. Am J Orthod Dentofac Orthop. 2005;127(2):200-7. quiz 260-201

9. Lundstrom A, McWilliam J. Comparison of some cephalometric distances and corresponding facial proportions with regard to heritability. Eur J Orthod. 1988;10(1):27-9.

10. Chai Y, Maxson RE Jr. Recent advances in craniofacial morphogenesis. Dev Dyn. 2006;235(9):2353-75.

11. Helms JA, Cordero D, Tapadia MD. New insights into craniofacial morphogenesis. Development. 2005;132(5):851-61.

12. Paternoster $L$, Zhurov Al, Toma AM, Kemp JP, St Pourcain B, Timpson NJ, McMahon G, McArdle W, Ring SM, Smith GD, et al. Genome-wide association study of three-dimensional facial morphology identifies a variant in PAX3 associated with nasion position. Am J Hum Genet. 2012;90(3):478-85

13. Liu F, van der Lijn F, Schurmann C, Zhu G, Chakravarty MM, Hysi PG, Wollstein A, Lao O, de Bruijne M, Ikram MA, et al. A genome-wide association study identifies five loci influencing facial morphology in Europeans. PLoS Genet. 2012;8(9):e1002932

14. Adhikari K, Fuentes-Guajardo M, Quinto-Sanchez M, Mendoza-Revilla J, Camilo Chacon-Duque J, Acuna-Alonzo V, Jaramillo C, Arias W, Lozano RB, Perez GM, et al. A genome-wide association scan implicates DCHS2, RUNX2, GLI3, PAX1 and EDAR in human facial variation. Nat Commun. 2016;7:11616.

15. Shaffer JR, Orlova E, Lee MK, Leslie EJ, Raffensperger ZD, Heike CL, Cunningham ML, Hecht JT, Kau CH, Nidey NL, et al. Genome-wide association study reveals multiple loci influencing normal human facial morphology. PLoS Genet. 2016;12(8):e1006149.

16. Cole JB, Manyama M, Kimwaga E, Mathayo J, Larson JR, Liberton DK, Lukowiak K, Ferrara TM, Riccardi SL, Li M, et al. Genomewide association study of African children identifies association of SCHIP1 and PDE8A with facial size and shape. PLoS Genet. 2016;12(8):e1006174.

17. Lee MK, Shaffer JR, Leslie EJ, Orlova E, Carlson JC, Feingold E, Marazita ML, Weinberg SM. Genome-wide association study of facial morphology reveals novel associations with FREM1 and PARK2. PLoS One. 2017;12(4):e0176566.

18. Nam J, Jang JS, Kim H, Kim JY, Do JH. Modification of the Integrated Sasang Constitutional Diagnostic Model. Evid Based Complement Alternat Med. 2017;2017:9180159

19. Ward LD, Kellis M. HaploReg v4: systematic mining of putative causal variants, cell types, regulators and target genes for human complex traits and disease. Nucleic Acids Res. 2016:44(D1):D877-81.

20. Machiela MJ, Chanock SJ. LDlink: a web-based application for exploring population-specific haplotype structure and linking correlated alleles of possible functional variants. Bioinformatics. 2015;31(21):3555-7.
21. Pickrell JK, Berisa T, Liu JZ, Segurel L, Tung JY, Hinds DA. Detection and interpretation of shared genetic influences on 42 human traits. Nat Genet. 2016;48(7):709-17.

22. Shaffer JR, Li J, Lee MK, Roosenboom J, Orlova E, Adhikari K, Gallo C, Poletti G, Schuler-Faccini L, Bortolini MC, et al. Multiethnic GWAS reveals polygenic architecture of earlobe attachment. Am J Hum Genet. 2017;101(6):913-24.

23. Claes P, Roosenboom J, White JD, Swigut T, Sero D, Li J, Lee MK, Zaidi A, Mattern BC, Liebowitz C, et al. Genome-wide mapping of global-to-local genetic effects on human facial shape. Nat Genet. 2018;50(3):414-23.

24. Tommerup N, Schempp W, Meinecke P, Pedersen S, Bolund L, Brandt C, Goodpasture C, Guldberg P, Held KR, Reinwein H, et al. Assignment of an autosomal sex reversal locus (SRA1) and campomelic dysplasia (CMPD1) to 17q24.3-q25.1. Nat Genet. 1993:4(2):170-4.

25. Mansour S, Offiah AC, McDowall S, Sim P, Tolmie J, Hall C. The phenotype of survivors of campomelic dysplasia. J Med Genet. 2002;39(8):597-602.

26. Smith TF. Diversity of WD-repeat proteins. Subcell Biochem. 2008;48:20-30.

27. Lin AE, Traum AZ, Sahai I, Keppler-Noreuil K, Kukolich MK, Adam MP, Westra SJ, Arts HH. Sensenbrenner syndrome (Cranioectodermal dysplasia): clinical and molecular analyses of 39 patients including two new patients. Am J Med Genet A. 2013;161A(11):2762-76.

28. Tsagkrasoulis D, Hysi P, Spector T, Montana G. Heritability maps of human face morphology through large-scale automated three-dimensional phenotyping. Sci Rep. 2017;7:45885

29. Gordon CT, Tan TY, Benko S, Fitzpatrick D, Lyonnet S, Farlie PG. Long-range regulation at the SOX9 locus in development and disease. J Med Genet. 2009:46(10):649-56

30. Leipoldt M, Erdel M, Bien-Willner GA, Smyk M, Theurl M, Yatsenko SA, Lupski JR, Lane AH, Shanske AL, Stankiewicz P, et al. Two novel translocation breakpoints upstream of SOX9 define borders of the proximal and distal breakpoint cluster region in campomelic dysplasia. Clin Genet. 2007:71(1):67-75.

31. Benko S, Fantes JA, Amiel J, Kleinjan DJ, Thomas S, Ramsay J, Jamshidi N, Essafi A, Heaney S, Gordon CT, et al. Highly conserved non-coding elements on either side of SOX9 associated with Pierre Robin sequence. Nat Genet. 2009;41(3):359-64.

32. Jakobsen LP, Ullmann R, Christensen SB, Jensen KE, Molsted K, Henriksen KF, Hansen C, Knudsen MA, Larsen LA, Tommerup N, et al. Pierre Robin sequence may be caused by dysregulation of SOX9 and KCNJ2. J Med Genet. 2007:44(6):381-6.

33. Gordon CT, Attanasio C, Bhatia S, Benko S, Ansari M, Tan TY, Munnich A, Pennacchio LA, Abadie V, Temple IK, et al. Identification of novel craniofacial regulatory domains located far upstream of SOX9 and disrupted in Pierre Robin sequence. Hum Mutat. 2014;35(8):1011-20.

34. Cho YS, Go MJ, Kim YJ, Heo JY, Oh JH, Ban HJ, Yoon D, Lee MH, Kim DJ, Park $\mathrm{M}$, et al. A large-scale genome-wide association study of Asian populations uncovers genetic factors influencing eight quantitative traits. Nat Genet. 2009:41(5):527-34.

35. Do J-H, Ku B, Jang J-S, Kim H, Kim JY. Analysis of Sasang constitutional types using facial features with compensation for photographic distance. Integr Med Res. 2012;1(1):26-35.

36. Castrillon M, Deniz O, Guerra C, Hernandez M. ENCARA2: real-time detection of multiple faces at different resolutions in video streams. J Vis Commun Image R. 2007:18(2):130-40.

37. Viola P, Jones MJ. Robust real-time face detection. Int J Comput Vis. 2004; 57(2):137-54.

38. Lee BJ, Do J-H, Kim JY. A classification method of normal and overweight females based on facial features for automated medical applications. J Biomed Biotechnol. 2012;2012:9.

39. Do JH, Jang E, Ku B, Jang JS, Kim H, Kim JY. Development of an integrated Sasang constitution diagnosis method using face, body shape, voice, and questionnaire information. BMC Complement Altern Med. 2012;12:85.

40. Jeong $\mathrm{S}, \mathrm{Yu}$ H, Lee $Y$, Kim JY. SNP genotyping through the melting analysis of unlabelled oligonucleotide applied on dilute PCR amplicon. J Biotechnol. 2011;154(4):321-5.

41. Cha S, Yu H, Kim JY. Bone mineral density-associated polymorphisms are associated with obesity-related traits in Korean adults in a sex-dependent manner. PLoS One. 2012;7(12):e53013.

42. Purcell S, Neale B, Todd-Brown K, Thomas L, Ferreira MA, Bender D, Maller J, Sklar P, de Bakker PI, Daly MJ, et al. PLINK: a tool set for whole-genome association and population-based linkage analyses. Am J Hum Genet. 2007:81(3):559-75.

43. Pruim RJ, Welch RP, Sanna S, Teslovich TM, Chines PS, Gliedt TP, Boehnke M, Abecasis GR, Willer CJ. LocusZoom: regional visualization of genome-wide association scan results. Bioinformatics. 2010;26(18):2336-7. 
44. Willer CJ, Li Y, Abecasis GR. METAL: fast and efficient meta-analysis of genomewide association scans. Bioinformatics. 2010;26(17):2190-1.

45. Human genomics. The Genotype-Tissue Expression (GTEx) pilot analysis: multitissue gene regulation in humans. Science. 2015;348(6235):648-60.

46. Ramasamy A, Trabzuni D, Guelfi S, Varghese V, Smith C, Walker R, De T, Coin $L$, de Silva $R$, Cookson MR, et al. Genetic variability in the regulation of gene expression in ten regions of the human brain. Nat Neurosci. 2014;17(10) 1418-28.

47. Schadt EE, Molony C, Chudin E, Hao K, Yang X, Lum PY, Kasarskis A, Zhang B, Wang $S$, Suver $C$, et al. Mapping the genetic architecture of gene expression in human liver. PLoS Biol. 2008;6(5):e107.

48. Westra HJ, Peters MJ, Esko T, Yaghootkar H, Schurmann C, Kettunen J, Christiansen MW, Fairfax BP, Schramm K, Powell JE, et al. Systematic identification of trans eQTLs as putative drivers of known disease associations. Nat Genet. 2013:45(10):1238-43.

49. Fairfax BP, Humburg P, Makino S, Naranbhai V, Wong D, Lau E, Jostins L, Plant K, Andrews R, McGee C, et al. Innate immune activity conditions the effect of regulatory variants upon monocyte gene expression. Science. 2014; 343(6175):1246949

50. Boyle AP, Hong EL, Hariharan M, Cheng Y, Schaub MA, Kasowski M, Karczewski KJ, Park J, Hitz BC, Weng S, et al. Annotation of functional variation in personal genomes using RegulomeDB. Genome Res. 2012; 22(9):1790-7.

Ready to submit your research? Choose BMC and benefit from:

- fast, convenient online submission

- thorough peer review by experienced researchers in your field

- rapid publication on acceptance

- support for research data, including large and complex data types

- gold Open Access which fosters wider collaboration and increased citations

- maximum visibility for your research: over $100 \mathrm{M}$ website views per year

At BMC, research is always in progress.

Learn more biomedcentral.com/submissions 